\title{
Grenzen der Freiheit? Werner Haftmann und die frühen Kunstdebatten im Jahresring
}

\section{The limits of liberty? Werner Haftmann and the early art debates in the Jahresring}

\author{
Christian Drobe
}

\begin{abstract}
This study looks at how the early art debates in the Jahresring Annual shaped out. Werner Haftmann was the most prominent figure in the post-war art scene and used various media to spread his ideas. In a programmatic contribution he articulated his ideal of artistic freedom, while his contemporaries were still struggling with the challenge of abstraction. A dispute between Hans Sedlmayr and Arnold Gehlen, later published in the Jahresring Annual, symbolizes the most prominent discourse of the whole 1950s, a debate, which altered between the desire for freedom and the wish to find new boundaries for contemporary art.
\end{abstract}

\section{Keywords}

abstraction; Arnold Gehlen; conservatism; continuity; modernism; Werner Haftmann; Jahresring Annual; Federal Republic of Germany; Hans Sedlmayr 


\section{Einleitung}

Nach 1945 setzte sich in den westlichen Industrienationen nach und nach die Abstraktion als bestimmende Kunstrichtung durch - nicht erst mit Serge Guilbauts Arbeit zum Einfluss US-amerikanischer Kulturpolitik eine problematische Setzung. ${ }^{1}$ In den letzten Jahren gewann der Kampf zwischen westlicher Abstraktion und heimischen figurativen Strömungen bzw. dem sozialistischen Realismus im Osten neue Konturen, gerade mit Blick auf die individuellen Strategien der Künstler. ${ }^{2}$ Dabei kommt häufig die Kontinuität zwischen Vor- und Nachkriegszeit zu kurz. ${ }^{3}$ Das Wirken und Denken vieler Persönlichkeiten, die vor und nach dem Krieg künstlerisch tätig waren, lässt sich im Bereich des Konservativen verorten. Dieser Zusammenhang verdient nach wie vor stärkere Beachtung im Fach Kunstgeschichte. Als Anfang 2020 der NSDAP-Mitgliedsausweis des bekannten Kunstkritikers Werner Haftmann, nach 1945 einer der stärksten Förderer der Moderne und der Abstraktion, gefunden wurde, schlug dies hohe Wellen. ${ }^{4}$ In welcher Weise sich Haftmann schuldig gemacht hat, wird Gegenstand weiterer Debatten bleiben. Aus ideengeschichtlicher Sicht ändert dieser Befund wenig daran, dass er seine Prägung als Kunsthistoriker unmittelbar vor bzw. während der NS-Herrschaft erhalten hat. Sind es Aspekte des geistigen Klimas der 1930er Jahre - mitunter in problematischer Nähe, aber eben nicht identisch mit dem Nationalsozialismus -, die die Nachkriegszeit und auch Haftmanns Vermittlung der Abstraktion und Moderne prägten?5 In welcher Weise können dabei konservative neben fortschrittlichen Positionen existieren? Um diesen Fragen nachzugehen, steht hier erneut die schon ältere These im Zentrum, die eine Kontinuität zwischen Weimarer Republik und Nachkriegszeit sieht, mithin über die Epochengrenzen von 1933 und 1945 hinausgeht. ${ }^{6}$

Mit Hans Mommsens These vom langen Schatten der Weimarer Republik lässt sich Haftmanns Werdegang exemplarisch einordnen. Sie kann auch für die Ausrichtung des Periodikums Jahresring herangezogen werden, das an Publikationen der Zwischenkriegszeit wie der Europäischen Revue anknüpfte. Das Journal wurde ab 1954 von Joachim

1 Guilbaut, Serge (1983): How New York stole the Idea of Modern Art. Abstract Expressionism, Freedom, and the Cold War. Chicago: Chicago University Press.

2 Hier exemplarisch: Fastert, Sabine (2010): Spontaneität und Reflexion. Konzepte vom Künstler in der Bundesrepublik Deutschland von 1945 bis 1960. Berlin - München: Dt. Kunstverlag.

3 vgl. Peters, Olaf (2005): Vom schwarzen Seiltänzer. Max Beckmann zwischen Weimarer Republik und Exil. Berlin: Reimer, S. 64, 429.

4 vgl. dazu Rauterberg, Hanno (2020): Hüter des falschen Friedens. In: Die ZEIT 7. Trinks, Stefan (2020): Braun, abstrakt. Werner Haftmann in der NS-Zeit. In: FAZ, 4. 2. 2020. Die gesamte jüngere Diskussion, die auf eine Tagung zur documenta-Geschichte in Berlin zurückgeht, wurde zusammengefasst von Redmann, Mirl (2020): Das Flüstern der Fußnoten. Zu den NS-Biografien der documenta Gründer*innen*. In: documenta studien \#09, S. 1-20.

5 Schäfer, Hans Dieter (2009): Das gespaltene Bewusstsein. Vom Dritten Reich bis zu den langen Fünfziger Jahren. Erw. Neuausg. Göttingen: Wallstein.

6 Mommsen, Hans (1987): Der lange Schatten der untergehenden Republik. Zur Kontinuität politischer Denkhaltungen von der späten Weimarer zur frühen Bundesrepublik. In: Bracher, Karl-Dietrich - Funke, Manfred - Jacobsen, Hans-Adolf (hrsg.): Die Weimarer Republik 1918-1933. Politik, Wirtschaft, Gesellschaft. Bonn: Droste, S. 552-586. 
Moras, Rudolf de le Roi, Hermann Rinn und zunächst Erhard Göpel (ab der zweiten Ausgabe Ludwig Grote) herausgegeben. Es bot Haftmann eine Plattform, seine Vision der Moderne und den Vorrang der Abstraktion zu artikulieren. Gleichzeitig fand er hier ein geistiges Umfeld, in dem er diesen Anspruch in Anknüpfung an ältere Ideen formulieren konnte. Hier konnten sich neben konservativen Literaten und Denkern der Nachkriegszeit auch Kunstkritiker wie Carl Georg Heise oder Carl Linfert zu Fragen der neuen Kunst äußern. Wie wirkte sich das Nebeneinander politisch und künstlerisch unterschiedlich ausgerichteter Autoren und Kritiker im Bereich der Kunst aus? Konservative Dichter wie Ernst und Friedrich Georg Jünger, die schon vor dem Krieg aktiv waren, traten genauso wie Hans Egon Holthusen oder Rudolf Alexander Schröder in den frühen Ausgaben des Jahresrings in Erscheinung. Ihnen standen Beiträge von Ilse Aichinger, Ingeborg Bachmann oder Paul Celan gegenüber. Das Periodikum deckte im Umfeld der Gegenwartsliteratur ein breites Spektrum von Positionen ab, ohne erkennbar eine Richtung zu bevorzugen. Diese Vielfalt setzte sich in den Abschnitten zur bildenden Kunst fort.

Wie erklärt sich im konservativen Klima der frühen Bundesrepublik der Einsatz für die Abstraktion? Wie konnte Neues begonnen werden im Jahresring, einem Organ der Industrie? Nicht zuletzt unter dem Eindruck der neuesten Strömungen aus Amerika entwickelte sich in der BRD ein System staatlicher Kunstförderung, zu der auch der 1951 gegründete Bundesverband der deutschen Industrie (BDI) Wesentliches beitrug und dabei gezielt die Abstraktion förderte. ${ }^{7}$ Der Verband gründete einen eigenen ,Kulturkreis und unterstützte Künstler durch die Vergabe von Preisen und Stipendien. Er kaufte zudem gezielt abstrakte Bildwerke an und bündelte damit die Interessen einzelner Mäzene aus der Industrie. Zu dieser Strategie gehörte nicht zuletzt das Periodikum Jahresring.

Innerhalb dieses offenen Konzepts erwies sich der Kunstkritiker Werner Haftmann als besonders geeigneter Beiträger, da er es verstand, die neue Kunstform Abstraktion mit Rückgriffen auf die eigene Geschichte und Tradition zu beschreiben. So dominierten in den frühen Ausgaben des Jahresrings seine Beiträge die Abschnitte zur bildenden Kunst. Insgesamt vier Artikel erscheinen zwischen 1954 und 1959. Den Auftakt bildete im ersten Jahrgang 1954 ein kurzer Beitrag zu Georg Meistermann und Fritz Winter. ${ }^{8}$ Für den Kunstkritiker deutsche Vorzeigekünstler, da sie schon vor dem Krieg der Abstraktion anhingen - zwei Künstler, die in den aktuellen Debatten die Kontinuität zur Zwischenkriegszeit herstellten. Die zentrale Veröffentlichung Haftmanns im Jahresring erfolgte jedoch 1957/58 und beschäftigte sich mit dem Thema der Modernen Kunst und Ihrer ,Politischen Idee ${ }^{9}$. Sie soll hier kurz zusammengefasst und daraufhin analysiert werden, wie Haftmann das politische Erbe der Moderne antritt, also den progressiven und mitunter avantgardistischen Strömungen, die vom frühen 20. Jahrhundert bis zum Beginn der NS-Herrschaft aktiv waren. Nach den Ereignissen des Ungarischen Volksaufstands 1956 widmete sich der gesamte folgende Jahrgang des Jahresrings dem Problem

7 vgl. Weiland, Anna (2017): Private Kunst- und Kulturförderung in der Bundesrepublik Deutschland. Dissertation, Univ. Heidelberg, nicht publiziert, S. 79-89.

8 Haftmann, Werner (1954): Georg Meistermann und Fritz Winter. In: Jahresring, S. 143-149.

9 Haftmann, Werner (1957/58): Moderne Kunst und Ihre „Politische Idee‘. In: Jahresring, S. 69-84, S. 71. 
der Freiheit. Haftmann stellte Überlegungen zur Korrumpierbarkeit von Kunst in den großen Diktaturen des 20. Jahrhunderts an. Was bedeutet gegenwärtig die Autonomie und Freiheit der Kunst? Auf welche Traditionen lässt sich in Krisenzeiten aufbauen? Die der Moderne? Die des romantischen Künstler-Genies? Und daran anknüpfend, inwiefern steht die Kontinuität älterer Konzepte in den Kunstbeiträgen exemplarisch für die Ausrichtung des Jahresrings?

In der Forschung erfuhr dieser Transfer älterer kunstästhetischer Konzepte im Rahmen des neuen Kulturauftrags Abstraktion schon zeitgenössisch Aufmerksamkeit und wurde ideengeschichtlich rekonstruiert. ${ }^{10}$ Für Klaus Lankheit lagen die Wurzeln des neuen Stils in der Romantik. Die absoluten Setzungen des romantischen Genies, philosophisch um 1800 durch den Idealismus legitimiert und damit befreit von den Zwängen der Realität, zeitigten Fragen nach der Verantwortung des Künstlers. Überlegungen zur Autonomie der Kunst und der Verantwortung in der Gesellschaft grundierten allgemein die Entstehung der Moderne. Die ein Jahr später im Jahresring aufgenommene Kunst-Debatte zwischen Hans Sedlmayr und Arnold Gehlen steht in dieser Tradition und zeigt, dass in den 1950er Jahren komplementär zum Freiheitsgedanken immer auch die Grenzen der Kunst verhandelt werden. Angesichts der Abstraktion als dominante Kunstrichtung bestanden große Zweifel, wohin diese entfesselte gestalterische Freiheit führen würde. Sedlmayrs Verlust der Mitte prägte die gesamten 1950er Jahre und zeugt von dem Versuch, das Phänomen zu verstehen und einzuhegen. ${ }^{11}$ Ablehnung und Unverständnis trafen auf Begeisterung und Hoffnung. In diesem Umfeld bewegte sich Haftmann. Gegen Ende der 1950er Jahre versucht er im Jahresring den Diskussionen um die Grenzen der Kunst mit dem Postulat der Freiheit zu begegnen.

\section{Werner Haftmann, Moderne Kunst und Ihre ,Politische Idee' (1957/58)}

Der Aufsatz bezieht sich unmittelbar auf den Ungarischen Volksaufstand von 1956 und nimmt ihn zum Anlass die Unterdrückung der modernen Kunst unter Goebbels, Lenin, Stalin und Mao Zedong zu thematisieren. Diese hatten in ihren diktatorischen Systemen mehr oder weniger stark restriktiv auf die Kunstentwicklung gewirkt. Ideologisch unterschiedlich motiviert geriet dabei zumeist die künstlerische Moderne ins Visier. Deren Vertreter wurden öffentlich angegriffen, Werke wurden beschlagnahmt, oder Künstler verfolgt und festgehalten. Die Ausstellung Entartete Kunst in München 1937 gilt als Fanal für die politische Unterdrückung der Kunst im Zeitalter der Extreme. Nach dem Ende des Krieges war es ein großes Anliegen die dort gezeigten Künstler wieder zu rehabili-

10 Lankheit, Klaus (1951): Die Frühromantik und die Grundlagen der „gegenstandslosen“ Malerei. In: Neue Heidelberger Jahrbücher, S. 55-90. Seine Ideen wurden später aufgenommen von: Rosenblum, Robert (1983): Modern painting and the northern romantic tradition. Friedrich to Rothko. Repr. der Ausg. von 1978. London: Thames and Hudson.

11 vgl. zuletzt zu Sedlmayr: Männig, Maria (2017): Hans Sedlmayrs Kunstgeschichte. Eine kritische Studie. Köln - Weimar - Berlin: Böhlau. 
tieren. Haftmann gehörte zu einem großen Befürworter dieser Idee. Der Restitutionsund Wiedergutmachungsgedanke gegenüber den verfolgten Künstlern sollte als Geste wirken, um wieder als freiheitliches Land wahrgenommen werden zu können. Den Ursprung des Freiheitsgedankens verortete Haftmann wiederum direkt in den politischen Ideen der Moderne selbst. So erklärt sich der Titel und Anlass des Aufsatzes. Es ging um die Verteidigung und historische Legitimierung der Freiheit (der Kunst) angesichts der Bedrohung durch neue diktatorische Systeme. Haftmanns Beitrag gliedert sich in vier Abschnitte: die gegenwärtige Lage unter dem Einfluss der Diktaturen, die politische Idee des Künstlers, das Aufkommen einer Weltkultur, und das Regionale im Globalen. $\mathrm{Zu}$ Beginn veranschaulicht Haftmann seine Auffassung wie folgt:

„Tatsächlich ist die in der modernen Kunst so unbedingt wirkende Idee der schöpferischen Freiheit und der dem einzelnen aufgetragenen Wirklichkeitsbestimmung ein gewaltiges politisches Agens. Vergleichbar einer Mikrobe, dringt sie in die geistigen Gewebe ein und verändert die emotionale und intellektuelle Struktur des Menschen und das ihn umgebende geistige Klima."12

Die Kunst trägt laut Haftmann den Gedanken der Freiheit in die Gesellschaften. Damit kann gegenwärtigen diktatorischen Systemen entgegengetreten werden. Unter dem Eindruck seines Aufenthalts bei der Biennale im Frühjahr 1956 und angesichts vieler Kontakte in den Osten Europas sah er eine große Gemeinschaft kommen, ein Kollektiv junger Maler, Schriftsteller und Kritiker, die den Ideen der Moderne folgen würden. Paternalistisch bemängelt er den kollektiven Geist der osteuropäischen Künstlergruppen, die noch das heimische und $d$. h. zumeist sozialistische Weltbild in sich tragen würden. Bald würden sie sich zu freiheitlich-westlichen, d. h. autonomen Künstler-Individuen weiterentwickeln und die Fesseln der kommunistischen Regime abschütteln - so Haftmanns Wunschvorstellung. Die Moderne westlicher Prägung wird zum Vorbild für den Kampf gegen Diktaturen. Naturgemäß sahen solche Emanzipationsbestrebungen durch die Niederschlagung des ungarischen Aufstandes im Oktober 1956 einem jähen Ende entgegen. Haftmann befürchtete, dass die in Ungarn gerade erst einsetzenden Lockerungen, die das Kunstgeschehen wie allerorts im Ostblock nach dem Tode Stalins beflügelten, wieder zurückgenommen werden würden.

Nach der Gegenwartsanalyse folgt der Gang durch die Geschichte. Welche Künstler hatten bisher eine politische Idee verfolgt? Die Beispiele politisch aktiver Künstler reichen von Camille Pissarro und Wassily Kandinsky bis zu George Grosz, Otto Dix oder Pablo Picasso - allesamt Vertreter der Moderne, die teils dann in der Zwischenkriegszeit besonders erfolgreich waren. Im Lichte der Ereignisse in Ungarn führt Haftmann vor, wie sich die Künstler dieser Generation langsam von den linken Vorbildern gelöst hätten. Angesichts der diktatorischen Praktiken und dem Machtwillen der kommunistischen Partei bzw. der Sowjetunion, der sich schon in den 1920er und 1930er Jahren zeigte, hätten sich viele von den verheißungsvollen Ideen des Sozialismus abgewandt. So kann

12 Haftmann [Anm. 8], S. 71. 
Haftmann bekräftigen, dass der moderne Künstler seine Heimat allenfalls in den westlichen Demokratien finden könne. Diesen stehen sie nicht uneingeschränkt positiv gegenüber, vielmehr komme ihnen dort eine wichtige, nahezu aufwieglerische Aufgabe zu. Sie richtet sich „gegen die nivellierten Gruppenanschauungen, das Übergewicht des Mittelmaßes durch die größere Zahl und den geistigen Konformismus. "13 Die politische Idee der Freiheit sei laut Haftmann auch im Westen noch nicht verwirklicht, sondern es sei geboten, sie in Verantwortung gegenüber dem einzelnen Individuum zu erstreiten. Die Kunst fungiere dort als individualistisch-elitäre Einrichtung gegen die Nivellierungstendenzen der demokratischen Massengesellschaften. Haftmann berührt damit einen Aspekt des antidemokratischen bzw. konservativen Denkens, der besonders in den 1920er Jahren populär war und in deren Zentrum der Gedanke geistiger Führerschaft steht. Schon Max Weber hatte die Charakteristika und Gefahren charismatischer Herrschaft analysiert. Im ungeliebten politischen System der Weimarer Republik waren viele der Meinung, dass man nur durch einen geeigneten ,Führer‘ den Problemen der Zeit Herr werden könne. Haftmanns Gedanken erinnern hier ebenfalls an Künstler wie Wassily Kandinsky oder Max Beckmann, die zu Beginn des 20. Jahrhunderts Vorstellungen der romantischen Kunstreligion aufgreifen und diese zur Vorstellung einer elitären Künstlerkaste ausformulieren. Gerade Beckmanns Ideen geistigen Adels knüpfen an Gedanken eines elitären Konservativismus an. ${ }^{14}$ Wieder werden Konzepte aus der Zeit der Weimarer Republik und davor aufgegriffen, so wie es laut Mommsen charakteristisch für die Nachkriegszeit ist.

In einem dritten Schritt beschreibt Haftmann die Ausweitung des zeitlichen und räumlichen Bewusstseins des Menschen: „[D]urch die moderne Nachrichten- und Vervielfältigungsmittel, durch die globale Verflechtung von Wirtschaft, Technik und Verkehr ist der ganze Erdkreis heute in Bezug gesetzt zur Lebens- und Bewußtseinshaltung des einzelnen."15 Dieser Akt sei laut Haftmann aber geistig und gefühlsmäßig von den Menschen noch nicht nachvollzogen worden. Es gelte, ein Vademekum gegen das Primat der Naturwissenschaften zu finden, so dass das Emotionale und Humanitäre nicht verlorengehe. Diese vereinheitlichte Weltkultur, so mangelhaft sie sei, sei wesentlich von Künstlern der Moderne vorangetrieben worden. Sie wirkten vorbildhaft und stilbildend um den ganzen Erdball: „Picasso, Matisse, Klee, Kandinsky, Mondrian sind heute im wörtlichen Sinne ,Weltmeister"."In der Architektur verbreitete sich mit dem Bauhaus und Le Corbusier auch der internationale Stil rasend schnell. Haftmanns These kulminiert in einem Perspektivwechsel: Solche Auswüchse von Regionalkulturen - so fasst er jetzt die Vertreter der Moderne zusammen -, wurden zur Vision einer Weltkultur. Sie erhielten allgemeine Gültigkeit. Mit der Zuordnung zum Regionalen gibt er der Moderne ein zeitliches Davor. Er erinnert wieder an ihre nationalen Wurzeln. Deutschland trug

13 Haftmann [Anm. 8], S. 76.

14 Beckmann, Max (1927): Der Künstler im Staat. In: Europäische Revue, S. 288-291. Zu Beckmanns zentralem Aufsatz, vgl. Peters [Anm. 3], S. 89-92. Beckmann lehnte aber den Nationalismus der dortigen Konservativen ab und formulierte ein politisch schwer einzuordnendes künstlerisches Programm, vgl. Peters [Anm. 3], S. 103-109.

15 Haftmann [Anm. 8], S. 77.

16 Haftmann [Anm. 8], S. 78. 
laut Haftmann etwa mit Klee und Kandinsky zu dieser Vision bei, Klee mit der bildenden Wirkkraft intuitiver Erkenntnisse, Kandinsky mit der Botschaft der abstrakten Malerei, als Ausdruck innerer Erfahrungen. Später fanden sie in der ganzen Welt Resonanz.

Ihm gelingt es so durch die Hintertür des Regionalen das Denken in nationalen Charakteristika am Leben zu erhalten. Im weiteren Verlauf des Artikels versucht er diese Idee zu untermauern. Mit Gemälden von Henri Matisse, Georges Braque und Fernand Léger führt er die vermeintliche Eigenheit französischer Kunst vor, die Erzeugung einer evokativen Fläche. Damit bezeichnet Haftmann wohlkonstruierte Gemälde, auf denen sowohl die einzelnen Bildelemente wie auch die gesamte Bildfläche harmonisch wirken. Sie erzeugen eine neue Harmonie parallel zur Natur. Die Deutschen verzichten laut Haftmann hingegen auf diese ausgewogene Formung. Beispiele von Ernst Ludwig Kirchner, Franz Marc und Oskar Schlemmer führen die Vorliebe der Deutschen für Geist, Emotionalität und Tiefe vor. Der Inhalt bestimmt die Komposition und illustriert die Werke. Keine Harmonie im französischen Sinne, so Haftmann, der damit an den alten Widerstreit zwischen französischer civilisation und deutscher Kultur anknüpft. Erneut ein altes Konzept, der Wettstreit geht aber jetzt versöhnlich aus.

Das System dieser Nationaltypen, meist orientiert an den Himmelsrichtungen - nordische Deutsche vs. mediterrane Franzosen -, gehörte bis in die Zwischenkriegszeit zum gängigen Repertoire der Kunsthistoriker. ${ }^{17}$ Haftmann knüpft damit an ein traditionelles kunsthistorisches Konzept an, das das konservative geistige Klima der 1920er und 1930 er Jahre in sich trägt. ${ }^{18}$ Die sogenannte Kunstgeografie war besonders bei Kunsthistorikern wie Josef Strzygowski beliebt. Jetzt soll sie unter anderen Vorzeichen den föderalistischen Charakter der globalisierten Weltgesellschaft untermalen. Der Schatten der Weimarer Republik wirkt auch hier. Einheit in der Vielheit, ein wichtiges Credo Haftmanns, das er seit den frühen Aufsätzen der 1930er Jahre in der Zeitschrift Kunst der Nation vertrat. ${ }^{19}$ Wenn er dort als junger Kunsthistoriker über die Geschichte der Kunst schrieb, zeichnete er ein möglichst vielschichtiges Bild der Vergangenheit. Keine Epoche ließ sich für Haftmann auf einen Kunststil reduzieren. Immer existierten mehrere Strömungen gleichzeitig nebeneinander. An diesem Anspruch hält er nach 1945 fest. So verbinden sich traditionelle und fortschrittliche Ideen.

Insgesamt führt Haftmann im Artikel Moderne Kunst und Ihre ,Politische Idee die Genese der regionalen zur internationalen Moderne vor, die für ihn nach 1945 weltweit Vorbildcharakter erhält. Wie in einer Kapsel trägt sie die politischen Kämpfe des frühen 20. Jahrhunderts in sich. Mit ihren nationalen Eigenheiten werden die Vertreter der klassischen Moderne nach 1945 so zu Botschaftern der Freiheit - jetzt als vom Kunstkritiker

17 Zur Logik der Himmelsrichtungen bei Haftmann vgl. Drobe, Christian (2020): Von Norden nach Westen: Werner Haftmann und Ernst Wilhelm Nay. In: Ernst Wilhelm Nay Stiftung (hrsg.): Ernst Wilhelm Nay und die Moderne. Köln: Walther König, S. 168-185.

18 Larsson, Lars Olof (1985): Nationalstil und Nationalismus in der Kunstgeschichte der zwanziger und dreiBiger Jahre. In: Dittmann, Lorenz (hrsg.): Kategorien und Methoden der deutschen Kunstgeschichte 19001930. Stuttgart: Steiner, S. 169-184.

19 vgl. dazu Germer, Stefan (1990): Kunst der Nation. Zu einem Versuch, die Avantgarde zu nationalisieren. In: Brock, Bazon - Preiß, Achim (hrsg.): Kunst auf Befehl? Dreiunddreißig bis Fünfundvierzig. München: Klinkhardt \& Biermann, S. 21-40. 
empfohlene Vorkämpfer politischer Skepsis und gemäßigter Dissidenz, dem Rahmen westlicher Demokratien entsprechend.

\section{Das Erbe des Expressionismus}

Die Zeitschrift Kunst der Nation entstand 1933 im Richtungsstreit nach der sogenannten Machtergreifung. Es ging darum, welcher Kunststil den neuen Machthabern zuträglich sein sollte. Unter den Fraktionen fanden sich nicht nur systemtreue Positionen, sondern auch die der Moderne - in Gestalt des Expressionismus, der als nordische Kunst den Deutschen besonders nahe schien. Es ging erneut darum kunstgeografisch eine bestimmte Strömung als zugehörig zum Nationalstil zu identifizieren. Der Kunsthistoriker Alois Schardt bemühte sich beispielsweise Künstler wie Emil Nolde oder Ernst Ludwig Kirchner bei den neuen Machthabern zu legitimieren, indem er auf die Tradition der Romantik und Gotik verwies. Ihr Stil ließ sich für ihn direkt auf diese heimischen Vorbilder zurückführen. Mit diesem Notbehelf wurde im Nationalsozialismus der Richtungsstreit um die Akzeptanz der Moderne geführt. Spätestens 1934/35 wurde dieser offenen Diskussion durch eine Grundsatzentscheidung Hitlers ein Ende gesetzt. Für Hitler blieb der Stil als Auswuchs der modernen Großstadt inakzeptabel. Die Analysen von Werken Kirchners oder Franz Marcs gegen Ende des Aufsatzes zur Modernen Kunst und Ihrer ,Politischen Idee zeigen Haftmann als Verfechter des deutschen Expressionismus. Seine beiden anderen Beiträge im Jahresring artikulieren dieses Interesse erneut - wichtig für die Restitution der Moderne nach 1945 in Deutschland. In der Ausgabe des Jahres 1955/56 erscheint der Artikel Die Lithographien Emil Noldes. ${ }^{20}$ Der Beitrag speist sich aus der längeren Beschäftigung Haftmanns mit dem Künstler, die in den späten 1940er Jahren einsetzte, zuerst mit Publikationen zu den Holzschnitten und Radierungen, und 1958 in eine wichtige Monographie mündete. ${ }^{21}$ Haftmann gehört zu seinem frühen Apologeten und schreibt mit am Mythos des verfolgten Künstlers. ${ }^{22}$ Er zeichnet Nolde als einsamen, spät zur Reife kommenden Maler. Der Kunstkritiker verortet die Lithographien im Kontext der Brücke-Künstler sowie im Zusammenhang mit dem Einfluss Edvard Munchs und des Expressionismus. Haftmanns verzichtet in diesem klassisch kunsthistorischen Beitrag, die wesentlich aus der Werkschau und Beschreibung der Blätter besteht, auf eine direkte politische Deutung. Er deutet Noldes Graphik als chthonisch-schwer, erdnah, urtümlich und melancholisch, gewinnt diese Ansichten aber aus der formalen Analyse. Die Graphik sei wie Malerei zu Farbmassen komponiert und wirke nicht feinästig von der Linie bestimmt wie bei anderen Künstlern. Haftmann beschreibt die Lithographien Noldes als direkten Ausdruck seines Schicksals und orien-

20 Haftmann, Werner (1955/56): Die Lithographien Emil Noldes. In: Jahresring, S. 154-170.

21 Haftmann, Werner (1958): Emil Nolde. Köln: DuMont.

22 Die jüngsten Auseinandersetzungen um Nolde im Nationalsozialismus und seine Rezeption nach 1945 können hier nicht in Gänze nachgezeichnet werden. Vgl. Fulda, Bernhard (2019): Die Entstehung einer deutschen Nachkriegslegende. In: Fulda, Bernhard - Ring, Christian - Soika, Aya (hrsg.): Emil Nolde - eine deutsche Legende. München - London - New York: Prestel, S. 221-247. 
tiert sich damit am gängigen Künstlermythos. Formale Qualitäten und die Nähe zum Expressionismus bestimmen die Deutung der Lithographien.

Im Aufsatz Ein deutscher Kunsthändler in Amerika, erschienen im Jahresring 1958/59, würdigt Haftmann die Verbreitung des deutschen Expressionismus in Amerika, die dank einiger neuer Publikationen in den USA endlich gefruchtet habe. Er vergleicht die Kunst mit dem expressiven Stil der aktuellen Malergeneration um Jackson Pollock, Hans Hartung, Wols oder Willem de Kooning. Es geht um das Informel, aber besonders um den Abstrakten Expressionismus, denn in ihm spiegelt sich der Transfer zwischen Europa und den USA. Deutsche Kunsthändler und Museumsleute wie Wilhelm Reinhold Valentiner, Alexander Dorner und Alois Schardt trugen im Falle des Expressionismus früh zu dessen Verbreitung bei. Im Zentrum des Beitrags steht allerdings Curt Valentin, dessen Berliner Galerie in den 1930er Jahren Zuflucht der freien Kulturschaffenden unter den Nazis war und der 1936 in die Dependance der Buchholz-Galerie nach Amerika ging. Dort wurde er zum umtriebigen Vermittler für die Moderne aus Deutschland, eng vertraut mit den Künstlern, wie die abschließend wiedergegebenen Briefe zeigen. Haftmann selbst sah sich in dieser Rolle, darauf zielt der Beitrag indirekt, zumal er zusammen mit Alfred Hentzen 1957 die große Ausstellung German Art of the $20^{\text {th }}$ century im Museum of Modern Art in New York eröffnen konnte.

Gerade das letzte Beispiel unterstützt Haftmanns These von der Verbreitung lokaler Kunstströmungen zur globalen Kunstwelt. Der deutsche Expressionismus fand weltweit eine große Anhängerschaft. Er wurde zum Aushängeschild der eigenen nationalen Moderne. Die beiden Artikel unterstützen die These indirekt als Fallbeispiele. Mit diesen Beiträgen Haftmanns lässt sich auch die Kunstsparte in den Ausgaben des Jahresrings von 1954-58/59 insgesamt charakterisieren. Sie bildet meist eine Abteilung pro Ausgabe, umfasst also jeweils etwa drei bis vier Beiträge. Abseits der Herausforderungen der Abstraktion oder der politischen Welt widmen sich die kunsthistorischen Beiträge klassischen Werkbeschreibungen oder würdigen einzelne Künstler, versammeln aber auch Überlegungen zur Architektur sowie zum Theater- und Museumsbau. In der Regel gibt es pro Ausgabe einen Artikel, der sich programmatisch zur Kunst äußert. Mit prominenten Kunsthistorikern wie Erhard Göpel und später Ludwig Grote unter den Herausgebern versuchte das ansonsten literarisch geprägte Periodikum an den aktuellen Debatten der Kunstwelt teilzunehmen und auch eine visuelle Wirkung zu erzeugen. Im Schnitt versammeln die Ausgaben etwa 40-45 Abbildungen, in den ersten Kapiteln häufig allgemein aus der Kunstgeschichte oder der Moderne gegriffen, in späteren Abschnitten aus der deutschen Gegenwartskunst. Nur die kunsthistorischen Beiträge sind thematisch entsprechend der Inhalte bebildert. Die Werke stammen häufig aus den Stiftungen und Schenkungen einzelner Industrieller, die etwa Eingang in Kunstsammlungen wie dem Folkwang Museum gefunden haben.

Diese neue kooperative Form des Mäzenatentums nach 1945 bestimmt die frühe Bundesrepublik und auch den Jahresring als Periodikum des Bundesverbands der Industrie (BDI). ${ }^{23} 1952$ wurde in der Hamburger Kunsthalle die Ausstellung Die Industrie als

23 vgl. Weiland [Anm. 6], S. 79-89. Vgl. auch Frey, Manuel (1999): Macht und Moral des Schenkens. Staat und bürgerliche Mäzene vom späten 18. Jahrhundert bis zur Gegenwart. Berlin: Fannei \& Walz, S. $186-209$. 
Kunstmäzen gezeigt. ${ }^{24}$ Sicher kein Zufall ist es, dass die frühen Ausgaben des Jahresrings Beiträge zur Geschichte des Mäzenatentums enthielten. Hier angesichts der Industriellen von einer dirigistischen, post-nazistischen, auf die Umerziehung der Bevölkerung abzielenden Kulturpolitik zu sprechen, wie es Silke Wenk getan hat, erscheint aus heutiger Sicht abwegig. ${ }^{25}$ Aus dem Geist der Industriekultur, repräsentiert durch den BDI, sollte kein weiterer Auswuchs der Kulturindustrie à la Theodor Adorno werden, sondern Ziel war es, die Kunst entlang der Probleme der Zeit zu fördern. Dazu passte der deutsche Expressionismus, gerettet vor den Nationalsozialisten, und auch die abstrakte Kunst als Zeichen des weltweiten Aufbruchs nach dem Krieg und der Angliederung an den Westen. Haftmanns Aufsätze formulierten dieses Hineinwirken regionaler Kräfte ins Konzert der großen globalisierten Welt-Kunst. Dass sie an ältere Konzepte aus der Zwischenkriegszeit anknüpften, war dabei zweitrangig.

Als Teil der Diskussionskultur, und dabei wollte man die Weimarer Republik hinter sich lassen, formierte sich ein Netzwerk gegenseitiger Förderer, die die Kunstöffentlichkeit der Nachkriegszeit bestimmten. Carl Linfert oder Alfred Hentzen waren häufiger mit Artikeln vertreten, später Eduard Trier, aber auch Künstler wie Gerhard Marcks oder Ernst Wilhelm Nay. In der Ausgabe 1959/60 publiziert Werner Hofmann seinen einzigen Artikel im Jahresring. Der österreichische Kunsthistoriker, berühmt geworden als Direktor der Hamburger Kunsthalle, nahm im Merkur, der ebenfalls von Joachim Moras mitherausgegeben wurde, eine deutlich größere Rolle ein. Er bildete damit nicht nur eine Art Gegengewicht, sondern verdeutlicht auch den Möglichkeitsspielraum, den die Herausgeber innerhalb ihrer verschiedenen Periodika gewährten. Je nach Ausrichtung des Publikationsorgans wurden die Kunstkritiker ausgewählt, hatten aber auch Gelegenheit, selbstbestimmt auf die neuesten Kunstentwicklungen zu reagieren. Diskussionsforen wie die Darmstädter Gespräche boten einzelnen Beiträgern und Intellektuellen zusätzlich Raum, ihre Ideen zu entwickeln. Die Idee einer sich multiplizierenden Öffentlichkeit kann trotz der konservativen Stimmung als wesentlicher Faktor für das Gelingen der frühen Bundesrepublik bis zum Wirtschaftswunder gelten.

\section{Grenzen der Kunst. Die Debatte zwischen Sedlmayr und Gehlen}

In der Jahresring-Ausgabe 1959/60 erschien unter dem Titel Bis wohin ist Malen Kunst? ein von Carl Linfert moderierter Disput. ${ }^{26}$ Das Streitgespräch geht zurück auf ein Treffen am 19. März 1959 im Saal des Wallraf-Richartz-Museums zu Köln innerhalb der Dis-

24 Ausstellungskatalog (1952): Die Industrie als Kunstmäzen. Gemälde, Plastik, Kunstgewerbe gestiftet in d. dt. Museen. Vom 6. Mai bis 8. Juni 1952. Veranstaltet vom Kulturkreis im Bundesverband d. dt. Industrie. Hamburg: Hamburger Kunsthalle. vgl. dazu Weiland [Anm. 6], S. 80.

25 Wenk, Silke (1980): Der Kulturkreis im BDI und die Macht der Kunst. In: Ausstellungskatalog: Zwischen Krieg und Frieden. Gegenständliche und realistische Tendenzen in der Kunst nach 45. Frankfurter Kunstverein 24. Oktober bis 7. Dezember 1980. Berlin: Elefanten-Press-Verlag, S. 80-83.

26 Linfert, Carl - Gehlen, Arnold - Sedlmayr, Hans (1959/60): Bis wohin ist Malen Kunst? Bericht über einen Disput. In: Jahresring, S. 65-84. 
kussionsreihe Umstrittene Sachen, die der Westdeutsche Rundfunk jedes Jahr im Winter veranstaltet hat. Linfert, der in dieser Zeit für seine Radiosendungen bekannt war, kommentiert die beiden abgedruckten Redebeiträge und versucht am Ende, die anschlieBende Diskussion im Saal zusammenzufassen. Zu Beginn formuliert er das Thema der Diskussionsrunde, die Frage nach den Grenzen der Kunst. Grenzüberschreitungen, in Form neuer Ismen, gehören zur Eigenart moderner Kunst, und angesichts der aktuellen Entwicklungen, der Herausforderung der Abstraktion, fragt Linfert nach dem Zu-weitGehen der Kunst und der Notwendigkeit neuer normativer Grenzen. Ist die neueste Kunst überhaupt noch Kunst?

Zuerst erhielt Sedlmayr das Wort, der sogleich mit einem Bezug zu Werner Haftmann und seinem bekannten Buch Malerei im 20. Jahrhundert einsetzt. ${ }^{27}$ Moderne Kunst, also vornehmlich Surrealismus und Abstraktion, sei „an zwei Gesten fixiert (diese Worte sind von Haftmann), die nicht mehr Kunst sind." ${ }^{28}$ Auch mit der nächsten Bezugsperson, dem Kunsthistoriker Oskar Karpa, macht er deutlich: Immer geht es um die Grenzüberschreitung, die durch die Abstraktion in die Welt gesetzt wurde. Da die gegenstandslose Bildgestaltung eine zweite Wirklichkeit parallel zur Natur schaffe, und dergestalt nicht mehr Kunst sei, setze mit ihr eine vermeintliche Zeitenwende ein, ein systemischer Wandel in der Kunst. An sechs Fragen erläutert Sedlmayr nun die Fallstricke dieser Grenzüberschreitung. Nach einem historischen Abriss der frühen Abstraktion von Kasimir Malewitsch und Wassily Kandinsky bespricht er die Loslösung der Form aus den alten, inhaltlich geprägten Bedeutungszusammenhängen. Dazu arbeitet er vornehmlich mit Teil-Ganzes-Relationen. Malewitschs Schwarzes Quadrat „ist nicht mehr als Kunstwerk zu bezeichnen, weil es sinnlos und äußerst unpraktisch wäre, ein Element, aus dem Kunstwerke sich aufbauen, mit dem gleichen Namen zu benennen wie das Ganze, aus dem es herausgenommen ist. "29 Seine These zielt also darauf ab, dass solche Bildelemente wie schwarze Quadrate schon immer Teil der Kunst waren, etwa in einer nächtlichen Landschaft, nun aber vereinzelt abgebildet und damit zum eigenen Werk gemacht werden würden. Diesem Vorgang spricht er den Kunststatus ab. Das Teil eines alten Ganzen könne keinen eigenen Werkstatus für sich beanspruchen.

Sedlmayr nennt diese Formen nun Parakunst, da „diese Kunst die Verabsolutierung von ,Teilen“ oder Elementen der alten Kunst ist." ${ }^{30}$ Auch Begriffe wie Partial-Kunst erscheinen ihm als passend. Verantwortlich für das Entstehen dieser Formen macht er u. a. den Reinheitsgedanken der Moderne, der zu einem immer größeren Formpurismus geführt habe. Weltanschaulich kulturkonservativ gerät der Ausblick, wenn er nach der Funktion der Parakunst in der modernen Welt fragt:

„Diese Parakunst umgibt, im Bündnis mit der Technik, den Menschen mit einer Umwelt von Artefakten, in der er nirgendwo mehr einer Natur begegnet, höchstens noch einer denaturierten,

27 Haftmann, Werner (1954): Malerei im 20. Jahrhundert. München: Prestel.

28 Sedlmayr [Anm. 24], S. 67.

29 Sedlmayr [Anm. 24], S. 69.

30 Sedlmayr [Anm. 24], S. 71. 
technisch präparierten, sondern nur mehr den Schöpfungen seines eigenen Ich [...] Diese Parakunst ist die adäquateste Kunstgestalt für den Menschen, der sich als absolut autonom proklamiert hat und, um diese illusionäre Autonomie zu behaupten, sich gegen die Natur und die lebendige Tradition abschließen muß, indem er sich mit einer Welt umgibt, die ganz von ihm selbst gemacht ist. Erst in einer solchen selbstfabrizierten Umwelt ist er ganz frei.“31

Dem freischaffenden, autonomen Künstler, dem Ideal Werner Haftmanns, der sich in der Abstraktion als künstlerischer Weltsprache ganz verwirklichen kann, tritt hier das Bild des traditions- und naturvergessenen Menschen gegenüber, der sich nur um sich selbst dreht. Sedlmayr zufolge kommt er in diesem Moment zur Freiheit. Beide Modelle gleichen sich, nur bei Sedlmayr fällt die Diagnose der Gesellschaft negativ aus. Analog zur Technik entfremden die artifiziellen Gebilde der Kunst den Menschen von sich selbst. Es fehle die Ganzheitlichkeit. Gedanken Heideggers klingen hier an, die er u. a. 1953 auf der großen Münchner Konferenz Die Künste im technischen Zeitalter geäußert hat. ${ }^{32}$ Gegenwartskunst und Technik wurden im Verbund gesehen. Wie in den gesamten 1950er Jahren nahm Sedlmayr dabei die konservative Position ein, als lautstarker Gegner der Abstraktion und Verfechter der künstlerischen Tradition. Wie auch Carl Linfert im anschließenden kurzen Kommentar bemerkt, ist nur so der verurteilende Ton seiner Analyse zu verstehen.

Arnold Gehlen entwickelt seine Entgegnung in insgesamt fünf Punkten. Aktiv setzt er sich gegen Sedlmayrs Bild vom selbstgefälligen, autonomen Menschen zur Wehr, der in der neuen Kunst nur sich selbst reproduziere. Das gehört laut Gehlen in den Bereich der Weltanschauung, nicht zur Analyse der Gesellschaft. Im Gegenteil, der Mensch stehe unter großem Druck von Staat und Politik und suche sich deshalb Freiräume in der Kunst. Entscheidend ist für ihn jedoch im ersten Punkt die Frage nach der Innovationskraft der aktuellen Kunst, gerade nach den Neuerungen der Klassischen Moderne um 1910, also denen von Expressionismus, Kubismus und Abstraktion:

„Es ist unmöglich, das Publikum durch irgendeine Bilderfindung auch nur zu schockieren, schon die letzte sogenannte Revolution von der geometrisierenden Abstraktion weg zum Tachismus war eine rein kunstimmanente. Ich sage das auch den Künstlern, denn jetzt entsteht die Frage, was aus einer einst revolutionären Kunst wird, wenn sie gesiegt hat, dann droht nicht der Akademismus der Vergangenheit, sondern der der Zukunft."33

Sedlmayr hatte noch versucht, die Neuerungen um 1910 als absoluten Traditionsbruch zu skandalisieren. Gehlen artikuliert damit das Bewusstsein für die Endlichkeit der auf

31 Sedlmayr [Anm. 24], S. 72.

32 Heidegger, Martin (1956): Die Frage nach der Technik. In: Bayerische Akademie der Schönen Künste (hrsg.): Die Künste im technischen Zeitalter. Eine Vortragsreihe der Bayerischen Akademie der Schönen Künste. Darmstadt: Wiss. Buchgesellschaft, S. 48-72. vgl. zur Konferenz: Morat, Daniel (2007): Von der Tat zur Gelassenheit: konservatives Denken bei Martin Heidegger, Ernst Jünger und Friedrich Georg Jünger. 1920-1960 (2. Aufl.). Göttingen: Wallstein, S. 470-484.

33 Gehlen [Anm. 24], S. 75. 
stetiger Progression aufgebauten Kunstentwicklung. Die nächste sogenannte Grenzüberschreitung vermag vielleicht nicht mehr etwas wirklich revolutionär Neues hervorzubringen. Wie Helmuth Kiesel in Hinblick auf Haftmann und Gehlen herausgearbeitet hat, herrscht in den 1950er Jahren ein starkes Bewusstsein dafür, dass die zeitgenössische Kunst eine Grenze erreicht hat. ${ }^{34}$ Gehlen beschreibt, analog zu den schwarzen und damit letzten Bildern von Künstlern wie Ad Reinhardt, die Unmöglichkeit künstlerischer Progression und Innovation als Folge systemischer Veränderungen. Sedlmayr kommt zu einer ähnlichen Diagnose, macht aber den allgemeinen Werteverlust dafür verantwortlich. Gehlen wählt für diesen Prozess später den Begriff der kulturellen Kristallisation, einen von Vilfredo Pareto übernommenen Terminus, der als Epochendiagnose der posthistoire häufig bei seinen Gesellschaftsanalysen zum Einsatz kommt. ${ }^{35}$ Liegt diesem Gedanken des Stillstandes auch etwas Konservatives zugrunde oder folgt die Kunstwelt nach 1945 wirklich nicht mehr sich schnell ablösenden Kunst-Ismen? ${ }^{36}$

Im nächsten Abschnitt erläutert Gehlen solche Grenzüberschreitungen im Bezugssystem der Kunst aus dem historischen Blickwinkel, also etwa in den Niederlanden im 16. Jahrhundert oder der Renaissance und ihrem neuen Wirklichkeitssinn. Dabei baut er langsam seine eigene Argumentation auf, die konträr zu Sedlmayr stärker ontologisch fundiert ist. Für Gehlen liegt der Reiz der Abstraktion in der Artikulation nicht mehr sprachfähiger Bereiche des Inneren. Diese Qualität wirke innovativ und gehe über die bloß subjektive Kunst des Expressionismus hinaus. Er kritisiert im Anschluss nicht nur Sedlmayrs Wortschöpfung der Parakunst, weil die Isolation von einzelnen Formen und deren Ausdifferenzierung schon immer wichtige Aspekte der Kunst bildeten, sondern verneint auch vermeintlich tiefenpsychologische Lesarten abstrakter Bilder, die seinerzeit beliebt waren. Weder der Künstler noch der Betrachter wisse, welcher seelischen Schicht sich das Bild widme, noch werde irgendeiner irrationalen Intuition Raum gegeben. Gehlen behauptet:

„abstrakte Bilder sind dann gut, wenn sie das optisch ganz präzise Gefühl einer nicht artikulierbaren Bedeutung erzwingen. [...] Die Dichter ringen mit der verwässerten Sprache, die ihren Klang verloren hat, in uns allen steigt der sprachlose Rest, das Unaussagbare und Unbewältigte, an dieser Stelle operiert, vom Auge her, das gute abstrakte Bild.“37

Diese optische Bedeutungskraft, welche die der Sprache transzendiert, steht für Gehlen im Zentrum der neuen Kunst. Und wenig später, nach dem Disput mit Sedlmayr, im Jahr

34 vgl. Kiesel, Helmuth (2004): Geschichte der literarischen Moderne. Sprache, Ästhetik, Dichtung im zwanzigsten Jahrhundert. München: C. H. Beck, S. 290. Die Theorie Gehlens erinnert an die heißen und kalten Gesellschaften von Claude Lévi-Strauss.

35 vgl. Kiesel [Anm. 32], S. 290.

36 Diese Fragen können hier nicht in Gänze diskutiert werden. Die Idee des Stillstands findet sich in vielen heutigen Analysen, vgl. etwa: Rebentisch, Juliane (2013): Theorien der Gegenwartskunst zur Einführung. Hamburg: Junius-Verlag, S. 9-24.

37 Gehlen [Anm. 24], S. 79. 
1960, erscheint seine Monografie Zeit-Bilder, in der er diese Argumentation bündelt. ${ }^{38}$ Darin wird er die These von der Erklärungsbedürftigkeit der modernen Kunst entwickeln. Schon im Jahresring findet sich in der letzten Passage des Disputs die Aussage, dass keine Kunst ohne Theorie groß geworden sei. Die vorsprachliche Erkenntnisqualität abstrakter Kunst komplementiert Gehlen in seiner Theorie mit einer sprach-lastigen Erklärungs- und Deutungskunst. Das ist abermals ein Plädoyer für die Kunstkritik und die öffentliche Diskussion, die die BRD nach 1945 etablierte. Der Angst vor dem Ende der traditionellen Kunst, die Sedlmayr artikuliert, begegnet der Soziologe Gehlen mit dem Gedanken der Kristallisation, der Verbildlichung des Vorsprachlichen und dem unendlichen Kunstgespräch. Linfert gibt sich in der abschließenden Anmerkung, in dem er auch die anschließende Diskussion zusammenzufassen sucht, als konservativer Kunstkommentator zu erkennen. Er verurteilt den Materie-Zauber aktueller Kunstschaffender, und die bloße Effekthascherei. Damit steht er Sedlmayr näher als Gehlen. Eine Synthese aus beiden Positionen sucht er nicht.

\section{Schluss}

Haftmann prägt die frühen Kunstdebatten im Jahresring. Angesichts aktueller politischer Ereignisse wie dem Aufstand in Ungarn 1956 fühlt er sich berufen, seine Vorstellung politisch motivierter Kunst zu artikulieren. Hans Mommsens These vom langen Schatten der Weimarer Republik folgend, zeigte sich, dass der autonome Künstler, der aus sich heraus zur Freiheit gelangt, bei Haftmann wesentlich von älteren Konzepten der Widerständigkeit und Autonomie bestimmt ist. Damit wird die Moderne zum Modellfall politischer Kunst. Sein Bild des politisch aktiven Künstlers bindet traditionelle Vorstellungen an die neuen Kunstformen nach 1945. Gleichzeitig wünscht er sich den Fortschritt in Gestalt einer globalisierten Weltsprache der Kunst, die die einzelnen Regional- und Nationalkulturen in sich aufnimmt. Auch hier werden ältere Aspekte der Kunstgeografie zu etwas Neuem umfunktioniert. Die Verbindung von alt und neu kommt der Ausrichtung des Bundesverbands der deutschen Industrie nahe, der sich nach 1945 nach Europa und der westlichen Welt orientiert und dergestalt massiv als Kunstförderer aufgetreten ist. Erst als Haftmanns Idee einer abstrakten Weltsprache als innerer Ausdruck des genialen Künstlers zusehends dogmatischer wird, fällt er hinter die aktuellen Trends zurück. In den 1960er Jahren findet er kaum Verständnis für die Pop-Art. Die Grenzen der Kunst-Freiheit wurden von anderen verhandelt. Hans Sedlmayr, Arnold Gehlen und Carl Linfert reagieren in einem Disput auf die andauernde Herausforderung der Abstraktion. Die Grenzüberschreitung der neuen Form ruft konservative Reflexe hervor, bestätigt aber in Gestalt Gehlens auch die Notwendigkeit der fortgesetzten Reflexion im öffentlichen Kunstgespräch, einer neuen diskursiven Wirklichkeit, die die westdeutsche Öffentlichkeit nach 1945 so tief prägte.

38 Gehlen, Arnold (1960): Zeit-Bilder. Zur Soziologie und Ästhetik der modernen Malerei. Frankfurt a. M. [u. a.]: Athenäum-Verlag. 


\section{Quellen und Literatur}

Ausstellungskatalog (1952): Die Industrie als Kunstmäzen. Gemälde, Plastik, Kunstgewerbe gestiftet in d. dt. Museen. Vom 6. Mai bis 8. Juni 1952. Veranstaltet vom Kulturkreis im Bundesverband d. dt. Industrie. Hamburg: Hamburger Kunsthalle.

Beckmann, Max (1927): Der Künstler im Staat. In: Europäische Revue 3, S. 288-291.

Drobe, Christian (2020): Von Norden nach Westen: Werner Haftmann und Ernst Wilhelm Nay. In: Ernst Wilhelm Nay Stiftung (hrsg.): Ernst Wilhelm Nay und die Moderne. Köln: Walther König, S. 168-185.

Fastert, Sabine (2010): Spontaneität und Reflexion. Konzepte vom Künstler in der Bundesrepublik Deutschland von 1945 bis 1960. Berlin - München: Dt. Kunstverlag.

Fastert, Sabine (2008): „Ich habe als europäischer Historiker geschrieben über europäische Malerei“. Werner Haftmanns Prinzipien der Kunstbetrachtung. In: Albrecht, Stephan - Braesel, Michaela (hrsg.): Kunst, Geschichte, Wahrnehmung. Strukturen und Mechanismen von Wahrnehmungsstrategien. Berlin: Deutscher Kunstverlag, S. 311-325.

Fitzke, Kirsten (2015): Auf dem Weg zur documenta. Die Wochenzeit DIE ZEIT und ihr Autor Werner Haftmann spiegeln und gestalten Positionen bildender Kunst in Westdeutschland. In: Panzer, Gerhard - Völz, Franziska - Rehberg, Karl-Siegbert (hrsg.): Beziehungsanalysen. Bildende Künste in Westdeutschland nach 1945. Akteure, Institutionen, Ausstellungen und Kontexte. Wiesbaden: Springer, S. 151-171.

Frey, Manuel (1999): Macht und Moral des Schenkens. Staat und bürgerliche Mäzene vom späten 18. Jahrhundert bis zur Gegenwart. Berlin: Fannei \& Walz.

Fulda, Bernhard (2019): Die Entstehung einer deutschen Nachkriegslegende. In: Fulda, Bernhard - Ring, Christian - Soika, Aya (hrsg.): Emil Nolde - eine deutsche Legende. München - London - New York: Prestel, S. 221-247.

Gehlen, Arnold (1960): Zeit-Bilder. Zur Soziologie und Ästhetik der modernen Malerei. Frankfurt a. M. [u. a.]: Athenäum.

Germer, Stefan (1990): Kunst der Nation. Zu einem Versuch, die Avantgarde zu nationalisieren. In: Brock, Bazon - Preiß, Achim (hrsg.): Kunst auf Befehl? Dreiunddreißig bis Fünfundvierzig. München: Klinkhardt \& Biermann, S. 21-40.

Guilbaut, Serge (1983): How New York stole the Idea of Modern Art. Abstract Expressionism, Freedom, and the Cold War. Chicago: Chicago University Press.

Haftmann, Werner (1955/56): Die Lithographien Emil Noldes. In: Jahresring, S. 154-170.

Haftmann, Werner (1958/59): Ein deutscher Kunsthändler in Amerika. In: Jahresring, S. 175-188. Haftmann, Werner (1958): Emil Nolde. Köln: DuMont.

Haftmann, Werner (1954): Georg Meistermann und Fritz Winter. In: Jahresring, S. 143-149.

Haftmann, Werner (1954): Malerei im 20. Jahrhundert. München: Prestel.

Haftmann, Werner (1957/58): Moderne Kunst und Ihre ,Politische Idee‘. In: Jahresring, S. 69-84.

Heidegger, Martin (1956): Die Frage nach der Technik. In: Bayerische Akademie der Schönen Künste (hrsg.): Die Künste im technischen Zeitalter. Eine Vortragsreihe der Bayerischen Akademie der Schönen Künste. Darmstadt: Wiss. Buchgesellschaft, S. 48-72.

Kiesel, Helmuth (2004): Geschichte der literarischen Moderne. Sprache, Ästhetik, Dichtung im zwanzigsten Jahrhundert. München: C. H. Beck.

Lankheit, Klaus (1951): Die Frühromantik und die Grundlagen der „gegenstandslosen“ Malerei. In: Neue Heidelberger Jahrbücher, S. 55-90.

Larsson, Lars Olof (1985): Nationalstil und Nationalismus in der Kunstgeschichte der zwanziger 
und dreißiger Jahre. In: Dittmann, Lorenz (hrsg.): Kategorien und Methoden der deutschen Kunstgeschichte 1900-1930. Stuttgart: Steiner, S. 169-184.

Linfert, Carl - Gehlen, Arnold - Sedlmayr, Hans (1959/60): Bis wohin ist Malen Kunst? Bericht über einen Disput. In: Jahresring, S. 65-84.

Männig, Maria (2017): Hans Sedlmayrs Kunstgeschichte. Eine kritische Studie. Köln - Weimar Berlin: Böhlau.

Mommsen, Hans (1987): Der lange Schatten der untergehenden Republik. Zur Kontinuität politischer Denkhaltungen von der späten Weimarer zur frühen Bundesrepublik. In: Bracher, Karl-Dietrich - Funke, Manfred - Jacobsen, Hans-Adolf (hrsg.): Die Weimarer Republik 19181933. Politik, Wirtschaft, Gesellschaft. Bonn: Droste, S. 552-586.

Morat, Daniel (2007): Von der Tat zur Gelassenheit: konservatives Denken bei Martin Heidegger, Ernst Jünger und Friedrich Georg Jünger. 1920-1960 (2. Aufl.). Göttingen: Wallstein.

Moser, Thomas (2014): „Kunst [ist das], was bedeutende Künstler machen.“ Zur Differenzierung zwischen Tradition und Innovation in Werner Haftmanns Schaffen der 50er und 60er Jahre. In: Helikon. A Multidisciplinary Online Journal 3, S. 35-53.

Müller, Franziska (2016): Ernst Wilhelm Nay: Vom Gestaltwert der Farbe - Artistic Concepts and Cultural Policy in Postwar Germany. In: Wünsche, Isabel - Gronemeyer, Wiebke (hrsg.): Practices of Abstract Art. Between Anarchism and Appropriation. Cambridge, S. 179-195.

Peters, Olaf (2005): Vom schwarzen Seiltänzer. Max Beckmann zwischen Weimarer Republik und Exil. Berlin: Reimer.

Rauterberg, Hanno (2020): Hüter des falschen Friedens. In: Die ZEIT 7.

Rebentisch, Juliane (2013): Theorien der Gegenwartskunst zur Einführung. Hamburg: Junius-Verlag.

Redmann, Mirl (2020): Das Flüstern der Fußnoten. Zu den NS-Biografien der documenta Gründer*innen*. In: documenta studien \#09, S. 1-20.

Rosenblum, Robert (1983): Modern painting and the northern romantic tradition. Friedrich to Rothko. Repr. der Ausg. von 1978. London: Thames and Hudson.

Schäfer, Hans Dieter (2009): Das gespaltene Bewusstsein. Vom Dritten Reich bis zu den langen Fünfziger Jahren. Erw. Neuausg. Göttingen: Wallstein.

Trinks, Stefan (2020): Braun, abstrakt. Werner Haftmann in der NS-Zeit. In: FAZ, 4. 2. 2020.

Weiland, Anna (2017): Private Kunst- und Kulturförderung in der Bundesrepublik Deutschland. Dissertation, Univ. Heidelberg, nicht publiziert.

Wenk, Silke (1980): Der Kulturkreis im BDI und die Macht der Kunst. In: Ausstellungskatalog: Zwischen Krieg und Frieden. Gegenständliche und realistische Tendenzen in der Kunst nach 45. Frankfurter Kunstverein 24. Oktober bis 7. Dezember 1980. Berlin: Elefanten-Press-Verlag, S. $80-83$.

Dr. Phil. Christian Drobe / drobe@phil.muni.cz

Masarykova univerzita, Filozofická fakulta, Ústav germanistiky, nordistiky a nederlandistiky Arna Nováka 1, 60200 Brno, CZ 